\title{
Exhibiting cross-diffusion-induced patterns for reaction-diffusion systems on evolving domains and surfaces
}

\author{
A. Madzvamuse* \\ School of Mathematical and Physical Sciences, Department of Mathematics, University of Sussex, \\ Pevensey III, 5C15, Falmer, Brigton, BN1 9QH, England, UK \\ R. Barreira ${ }^{\dagger}$ \\ Escola Superior de Tecnologia do Barreiro/IPS, Rua Américo da Silva Marinho-Lavradio, 2839-001 Barreiro, Portugal
}

(Received 30 July 2014; published 31 October 2014)

\begin{abstract}
The aim of this manuscript is to present for the first time the application of the finite element method for solving reaction-diffusion systems with cross-diffusion on continuously evolving domains and surfaces. Furthermore we present pattern formation generated by the reaction-diffusion system with cross-diffusion on evolving domains and surfaces. A two-component reaction-diffusion system with linear cross-diffusion in both $u$ and $v$ is presented. The finite element method is based on the approximation of the domain or surface by a triangulated domain or surface consisting of a union of triangles. For surfaces, the vertices of the triangulation lie on the continuous surface. A finite element space of functions is then defined by taking the continuous functions which are linear affine on each simplex of the triangulated domain or surface. To demonstrate the role of cross-diffusion to the theory of pattern formation, we compute patterns with model kinetic parameter values that belong only to the cross-diffusion parameter space; these do not belong to the standard parameter space for classical reaction-diffusion systems. Numerical results exhibited show the robustness, flexibility, versatility, and generality of our methodology; the methodology can deal with complicated evolution laws of the domain and surface, and these include uniform isotropic and anisotropic growth profiles as well as those profiles driven by chemical concentrations residing in the domain or on the surface.
\end{abstract}

DOI: 10.1103/PhysRevE.90.043307

PACS number(s): 02.70.Dh, 02.40.Sf, 02.60.Cb, 02.30.Jr

\section{INTRODUCTION}

Understanding of biological processes during growth development is an unresolved issue in developmental biology that is only starting to be addressed recently. Introducing domain growth into the modeling results in nonautonomous systems of partial differential equations whose analytical tractability is not well understood [1-5]. In the area of developmental biology, partial differential equations for pattern formation take the form of the reaction-diffusion type [6,7]. On stationary domains, for example, it is well known that one major criticism of reaction-diffusion theory for pattern formation is the tight control of the model reaction kinetic parameter values [8]. Underpinning this theory is the concept of diffusion-driven instability, which leads to patterns that are stationary in time and periodic in space. For a two-component reaction-diffusion system, a key requirement for diffusion-driven instability is the concept of long-range inhibition and short-range activation [9]. This implies that one of the species (the inhibitor) must diffuse faster (typically much faster) than the autocatalytic species (activator) thereby fulfilling one of the necessary conditions for the formation of spatial structure.

Several generalizations of the reaction-diffusion theory for pattern formation have been undertaken in order to relax some of these constraints. One of these generalizations involve the introduction of domain growth [1,3-5]. It is well known

\footnotetext{
*Corresponding author: a.madzvamuse@ sussex.ac.uk

${ }^{\dagger}$ raquel.barreira@estbarreiro.ips.pt
}

that many problems in biology involve growth. In [5] we proved that in the presence of domain growth, it is no longer necessary to restrict reaction kinetics to an activator-inhibitor type; a long-range activation and short-range inhibition or activation chemical process are all capable of giving rise to what we termed domain-growth induced diffusion-driven instability. However, this generalization still requires that the inhibitor must diffuse much faster than the activator species, and therefore equal diffusion coefficients do not give rise to the formation of spatial structure during growth development.

Another generalization is the introduction of crossdiffusion. In many multicomponent systems, there are various forms of diffusion depending on the biochemical problem at hand [10]. Diffusive processes can be characterized as selfdiffusion, cross-diffusion, mutual diffusion, tracer diffusion, intradiffusion, interdiffusion, uphill diffusion, and negative or incongruent diffusion. A detailed review of the different physicochemical interpretations of these forms of diffusion is given by Vanag and Epstein [10]. Cross-diffusion is characterized by a gradient in the concentration of one species inducing a flux of another chemical species. In molecular biology, cross-diffusion processes appear in multicomponent systems containing at least two solute components [11,12]. Multicomponent systems containing nanoparticles, surfactants, polymers, and other macromolecules in solution play an important role in industrial applications and biological functions [11]. In developmental biology, recent experimental findings demonstrate that cross-diffusion can be quite significant in generating spatial structure [10]. The effects of cross-diffusion on models for pattern formation (i.e., reaction-diffusion type) have been studied in many theoretical 
papers [13-24]. Recently, in [25] we showed that introducing cross-diffusion to a system of reaction-diffusion equations results in further relaxation of the conditions necessary for the emergency of patterns. In particular, an inhibitor and activator or two activators can diffuse at equal rates; however, the product of the rates of the principal diffusion coefficients must be greater than the product of the cross-diffusion rates. For detailed theoretical analytical and computational results on the effects of domain growth on pattern formation, the interested reader is referred to results published in [1,2,4,5,26-29]. In this article our focus is to showcase how cross-diffusion induces patterning in the presence of domain and surface evolution.

Despite all these advances, all the above studies have been carried out on stationary domains and to the authors' knowledge no work has been carried out on surfaces. In this article we will introduce domain and surface evolution and solve reaction-cross-diffusion systems with uniform linear isotropic, anisotropic, and concentration-driven evolution profiles. In the last case, apart from solving for the unknown chemical species, we also solve for the unknown domain and surface position. On planar domains, a spring analogy is employed to move the internal bulk mesh.

Recently there has been an increase in the development of numerical methods for approximating solutions of partial differential equations posed on evolving domains and surfaces. Examples include (but are not limited to) the moving grid finite element method [4], the method of lines [30], evolving surface finite element methods on triangulated surfaces [31-33], implicit finite element methods using level set descriptions of the surfaces [34-37], diffuse interface methods of which phase fields are an example [38,39], particle methods using level set descriptions of the surface [40-42], and closest-point methods [27,28]. In this article we choose to implement the evolving surface finite element method. Our approach is based on the surface finite element method, which is a natural extension of the finite element method and is capable of handling complex geometries and shapes [32,34,35,39]. On evolving domains, given the boundary evolution law, the internal mesh is deformed using a spring analogy [4]. On evolving surfaces, we triangulate the surface and approximate the system of partial differential equations using piecewise linear surface finite element spaces based on the triangulation. In this paper we are interested in evolving domains and surfaces. In this instance the vertices of the triangulation are moved with a velocity which either is prescribed or is governed by some evolution law. In order to do this we need to formulate an appropriate conservation law on the surface. The evolving surface finite element method exploits the special features of this conservation law when written in an appropriate variational form.

Hence, our paper is organized as follows: in Sec. II we present the model equations posed on evolving domains and surfaces, and these consist of a system of reactiondiffusion equations with linear cross-diffusion. Domain and surface evolution terms are modeled through dilution and convective terms. The finite element numerical methodology is presented in Sec. III. The methodology is characterized by two fundamental steps: first, a spatial discretization is employed that exploits the conservative nature of the modeling to give rise to a system of ordinary differential equations. The second step involves the implementation of a modified backward Euler scheme to transform the system of ordinary differential equations into a system of linear algebraic equations amenable for computer simulations. Section IV details numerical experiments demonstrating the effects of the inclusion of crossdiffusion into the models for pattern formation. Here uniform isotropic, anisotropic, and concentration-driven evolution laws are considered to demonstrate the generality of the modeling and computational methodology. We select model parameter values that give rise to the formation of spatial structure only in the presence of cross-diffusion and compare our results to classical parameter values also in the presence of crossdiffusion. Such cross-diffusion-driven parameter values do not give rise to pattern formation in the absence of cross-diffusion. In Sec. $\mathrm{V}$ we conclude and discuss the implications of our findings to the theory and computations of models for pattern formation.

\section{MODELS POSED ON EVOLVING DOMAINS AND SURFACES}

\section{A. Notation and preliminaries}

In this section we establish some notation to be used throughout the paper.

If $\Gamma \subset \mathbb{R}^{3}$ is a two-dimensional hypersurface with unit normal $\boldsymbol{v}$ we denote the tangential gradient of a scalar function $u$ defined on $\Gamma$ which is differentiable in an open neighborhood of $\Gamma$ by

$$
\nabla_{\Gamma} u:=\nabla u-\nabla u \cdot v \boldsymbol{v},
$$

where $\nabla$ denotes the usual gradient in $\mathbb{R}^{3}$.

If $u$ is twice differentiable in an open neighborhood of $\Gamma$, we define the Laplace-Beltrami operator as the tangential divergence of the tangential gradient

$$
\Delta_{\Gamma} u:=\nabla_{\Gamma} \cdot \nabla_{\Gamma} u
$$

If $\Gamma(t)$ is a hypersurface in $\mathbb{R}^{3}$ evolving in time, $t$, according to a velocity field $v$, we denote the material derivative of $u$ by

$$
\partial^{\bullet} u:=u_{t}+\boldsymbol{v} \cdot \nabla u .
$$

\section{B. Reaction-diffusion systems with linear cross-diffusion posed on evolving domains and surfaces \\ 1. Reaction-diffusion system with linear cross-diffusion posed on evolving domains}

Let $\Omega(t) \subset \mathbb{R}^{m} \quad(m=1,2,3)$ be a simply connected bounded evolving volume for all time $t \in I=\left[0, t_{F}\right], t_{F}>$ 0 , and $\partial \Omega(t)$ be the evolving boundary enclosing $\Omega(t)$. Also let $\boldsymbol{u}=(u(\boldsymbol{x}, t), w(\boldsymbol{x}, t))^{T}$ be a vector of two chemical concentrations at position $x \in \Omega(t) \subset \mathbb{R}^{m}$. The evolution equations for reaction-diffusion systems with cross-diffusion can be obtained from the application of the law of mass conservation in an elemental volume using the Reynolds transport theorem. The growth of the volume $\Omega(t)$ generates a 
flow of velocity $v$ to yield the nondimensional system

$$
\left\{\begin{array}{l}
\left\{\begin{array}{l}
u_{t}+\nabla \cdot(\boldsymbol{v} u)=\nabla^{2} u+d_{w} \nabla^{2} w+\gamma f(u, w), \\
w_{t}+\nabla \cdot(\boldsymbol{v} w)=d \nabla^{2} w+d_{u} \nabla^{2} u+\gamma g(u, w),
\end{array}\right. \\
\boldsymbol{n} \cdot \nabla u=\boldsymbol{n} \cdot \nabla w=0, \quad x \text { on } \partial \Omega(t), \quad t \geqslant 0, \quad t \geqslant 0, \\
u(\boldsymbol{x}, 0)=u_{0}(\boldsymbol{x}), \quad \text { and } \quad w(\boldsymbol{x}, 0)=w_{0}(\boldsymbol{x}), \quad \boldsymbol{x} \text { on } \Omega(0),
\end{array}\right.
$$

where $\nabla^{2}$ is the Laplace operator on domains and volumes, $d$ is the ratio of the diffusion coefficients, and $d_{u}$ and $d_{w}$ are the ratios of the cross-diffusion. Here $\boldsymbol{n}$ is the unit outward normal to $\Omega(t)$. Initial conditions are prescribed through non-negative bounded functions $u_{0}(\boldsymbol{x})$ and $w_{0}(\boldsymbol{x})$. In the above, $f(u, w)$ and $g(u, w)$ represent nonlinear reactions.

\section{Reaction-diffusion system with linear cross-diffusion posed on evolving surfaces}

Supposing now the domain is an evolving hypersurface, $\Gamma(t)$, with velocity $\boldsymbol{v}=V \boldsymbol{v}+V_{T}$, where $\boldsymbol{v}$ is the unit outward pointing normal vector to $\Gamma(t), V$ is the normal velocity, and $V_{T}$ is the tangential velocity. Without loss of generality, let $\boldsymbol{u}=(u(\boldsymbol{s}, t), w(\boldsymbol{s}, t))^{T}$ be a vector of two chemical concentrations at position $\boldsymbol{s} \in \Gamma(t) \subset \mathbb{R}^{m+1}(m=1,2)$. Then adapting the derivation of the reaction-diffusion equations on an evolving surface in [31], taking into account the terms corresponding to cross-diffusion, we obtain the following nondimensionalized system of reaction-diffusion equations:

$$
\left\{\begin{array}{l}
\left\{\begin{array}{l}
u_{t}+\nabla_{\Gamma(t)} \cdot(\boldsymbol{v} u)=\nabla_{\Gamma(t)}^{2} u+d_{w} \nabla_{\Gamma(t)}^{2} w+\gamma f(u, w), \\
w_{t}+\nabla_{\Gamma(t)} \cdot(\boldsymbol{v} w)=d \nabla_{\Gamma(t)}^{2} w+d_{u} \nabla_{\Gamma(t)}^{2} u+\gamma g(u, w),
\end{array} \quad \boldsymbol{s} \in \Gamma(t), \quad t \geqslant 0,\right. \\
u(\boldsymbol{s}, 0)=u_{0}(\boldsymbol{s}), \quad \text { and } \quad w(\boldsymbol{s}, 0)=w_{0}(\boldsymbol{s}), \quad \boldsymbol{s} \text { on } \Gamma(0)
\end{array}\right.
$$

where we are assuming $\Gamma(t)$ is a compact smooth connected and oriented evolving hypersurface in $\mathbb{R}^{m+1}$, with $m=1,2$.

As for the boundary conditions, we impose $\boldsymbol{\mu} \cdot \nabla_{\Gamma(t)} u=$ $\boldsymbol{\mu} \cdot \nabla_{\Gamma(t)} w=0$, where $\boldsymbol{\mu}$ is the conormal vector to $\partial \Gamma(t)$, if $\Gamma(t)$ is nonempty. Otherwise, if the surface has no boundary, then there is no need for boundary conditions.

Remark 2.1. Notice that for the planar case the tangential derivative coincides with the classical derivative and there is no normal velocity in that case, so the model equations become exactly the same as (2.1).

Remark 2.2. In many articles in the literature, model systems (2.1) and (2.2) can be mapped at all times from a continuously deforming domain or surface to a reference static domain or surface for analytical tractability as well as for computational purposes $[1,2,4,5,26]$. This implies that analytical and computational results are scale invariant. In this work we will treat the model systems posed on a continuously evolving domain or surface.

\section{Activator-depleted reaction kinetics: An illustrative example}

For illustrative purposes, we consider a specific type of nonlinear reactions, namely, the activator-depleted model also known as the Brusselator model $[9,43,44]$ :

$$
f(u, w)=a-u+u^{2} w, \quad \text { and } \quad g(u, w)=b-u^{2} w,
$$

with $a, b \in \mathbb{R}^{+}$.

\section{NUMERICAL SOLUTION OF REACTION-DIFFUSION SYSTEMS WITH CROSS-DIFFUSION ON EVOLVING DOMAINS AND SURFACES}

In this section, we present the finite element method applied to reaction-diffusion systems on evolving domains and surfaces. The method becomes the moving grid finite element method on evolving planar domains [4,45] and the evolving surface finite element method on evolving surfaces [31]. The numerical methodology is equivalent for both cases, the only difference being in the spatial operators between planar domains and surfaces as well as the use of boundary conditions. To this end, we will simply derive a numerical method for solving (2.2) on a hypersurface and then treat a planar domain as a hypersurface with appropriate boundary conditions.

\section{A. The surface finite element method applied to reaction-cross-diffusion systems}

The model equations in (2.2) can be rewritten as

$$
\left\{\begin{array}{c}
\partial \bullet u+u \nabla_{\Gamma(t)} \cdot \boldsymbol{v}=\nabla_{\Gamma(t)}^{2} u+d_{w} \nabla_{\Gamma(t)}^{2} w+\gamma f(u, w), \\
\partial \bullet w+w \nabla_{\Gamma(t)} \cdot \boldsymbol{v}=d \nabla_{\Gamma(t)}^{2} w+d_{u} \nabla_{\Gamma(t)}^{2} u+\gamma g(u, w) .
\end{array}\right.
$$

The evolving surface finite element introduced in [32] has been already extended in [31] in order to solve the model equations for the reaction-diffusion systems, and the new cross-diffusion terms can be treated in a similar fashion and added to the existing numerical scheme. For the sake of completeness, we will present the derivation of the finite element method for the reaction-diffusion systems with cross-diffusion.

\section{Variational formulation}

The variational formulation for the first equation corresponding to the chemical specie $u$ in (3.1) will be presented as the other equation for $w$ can be treated in exactly the same fashion since both have similar structures. For ease of reading, we will omit the dependence of $t$ in the notation for the tangential gradient, so we will use $\nabla_{\Gamma}$ instead of $\nabla_{\Gamma(t)}$. 
Let $\varphi(\cdot, t) \in H^{1}(\Gamma(t))$ be a test function. Multiplying the equation by $\varphi$ and integrating over $\Gamma(t)$ leads to

$$
\begin{aligned}
\gamma \int_{\Gamma(t)} f \varphi= & \int_{\Gamma(t)} \partial \bullet u \varphi+\int_{\Gamma(t)} u \varphi \nabla_{\Gamma} \cdot \boldsymbol{v}-\int_{\Gamma(t)} \varphi \nabla_{\Gamma}^{2} u-d_{w} \int_{\Gamma(t)} \varphi \nabla_{\Gamma}^{2} w \\
= & \int_{\Gamma(t)} \partial^{\bullet} u \varphi+\int_{\Gamma(t)} u \varphi \nabla_{\Gamma} \cdot \boldsymbol{v}+\int_{\Gamma(t)} \nabla_{\Gamma} \varphi \cdot \nabla_{\Gamma} u-\int_{\partial \Gamma(t)} \varphi \nabla_{\Gamma} u \cdot \boldsymbol{\mu} \\
& +d_{w} \int_{\Gamma(t)} \nabla_{\Gamma} \varphi \cdot \nabla_{\Gamma} w-d_{w} \int_{\partial \Gamma(t)} \varphi \nabla_{\Gamma} u \cdot \boldsymbol{\mu} .
\end{aligned}
$$

If $\partial \Gamma(t)=\emptyset$ or if $\nabla_{\Gamma} u \cdot \boldsymbol{\mu}=0$, the two integrals over $\partial \Gamma(t)$ vanish. Assuming any of these conditions hold we have

$$
\begin{aligned}
\gamma \int_{\Gamma(t)} f \varphi & =\int_{\Gamma(t)} \partial^{\bullet} u \varphi+u \varphi \nabla_{\Gamma} \cdot \boldsymbol{v}+\int_{\Gamma(t)} \nabla_{\Gamma} u \cdot \nabla_{\Gamma} \varphi+d_{w} \int_{\Gamma(t)} \nabla_{\Gamma} w \cdot \nabla_{\Gamma} \varphi \\
& =\int_{\Gamma(t)} \partial^{\bullet}(u \varphi)-u \partial^{\bullet} \varphi+u \varphi \nabla_{\Gamma} \cdot v+\int_{\Gamma(t)} \varphi \nabla_{\Gamma} u \cdot \nabla_{\Gamma} \varphi+d_{w} \int_{\Gamma(t)} \nabla_{\Gamma} w \cdot \nabla_{\Gamma} \varphi \\
& =\frac{d}{d t} \int_{\Gamma(t)} u \varphi-\int_{\Gamma(t)} u \partial^{\bullet} \varphi+\int_{\Gamma(t)} \nabla_{\Gamma} u \cdot \nabla_{\Gamma} \varphi+d_{w} \int_{\Gamma(t)} \nabla_{\Gamma} w \cdot \nabla_{\Gamma} \varphi
\end{aligned}
$$

The variational form seeks to find $u \in H^{1}(\Gamma(t))$ satisfying

$$
\frac{d}{d t} \int_{\Gamma(t)} u \varphi-\int_{\Gamma(t)} u \partial^{\bullet} \varphi+\int_{\Gamma(t)} \nabla_{\Gamma} u \cdot \nabla_{\Gamma} \varphi+d_{w} \int_{\Gamma(t)} \nabla_{\Gamma} w \cdot \nabla_{\Gamma} \varphi=\gamma \int_{\Gamma(t)} f(u, w) \varphi,
$$

for all $\varphi \in H^{1}(\Gamma(t))$. Similarly, using the same reasoning for the second equation of (2.2), the variational form seeks to find $w \in H^{1}(\Gamma(t))$ satisfying

$$
\frac{d}{d t} \int_{\Gamma(t)} w \varphi-\int_{\Gamma(t)} w \partial^{\bullet} \varphi+\int_{\Gamma(t)} \nabla_{\Gamma} w \cdot \nabla_{\Gamma} \varphi+d_{u} \int_{\Gamma(t)} \nabla_{\Gamma} u \cdot \nabla_{\Gamma} \varphi=\gamma \int_{\Gamma(t)} g(u, w) \varphi,
$$

for all $\varphi \in H^{1}(\Gamma(t))$.

\section{B. Evolving surface finite element method (ESFEM)}

We approximate $\Gamma(t)$ by $\Gamma_{h}(t)$, a triangulated surface whose vertices lie on $\Gamma(t)$, i.e., $\Gamma_{h}(t)=\mathcal{T}_{h}(t)=\bigcup_{k} T_{k}(t)$, where each $T_{k}(t)$ is a triangle. The diameter of the largest triangle in the initial surface is denoted by $h$. We choose the vertices of the triangulation to evolve with the material velocity such that

$$
\dot{X}_{j}(t)=v\left(X_{j}(t), t\right)
$$

It is easy to note that $X_{j}(t)$ lies on $\Gamma(t)$ if $v$ is the exact material velocity. We assume $\Gamma_{h}(t)$ is smooth in time. For each $t$ we define a finite element space

$$
\begin{aligned}
S_{h}(t)= & \left\{\phi \in C^{0}\left(\Gamma_{h}(t)\right): \phi T_{k},\right. \\
& \text { is linear affine for each } \left.T_{k} \in \mathcal{T}_{h}(t)\right\} .
\end{aligned}
$$

For each $t \in\left[0, t_{F}\right]$ we denote by $\left\{\chi_{j}(\cdot, t)\right\}_{j=1}^{N}$ the moving nodal basis functions and by $X_{j}(t), j=1, \ldots, N$ the nodes. These functions will satisfy that

$$
\begin{aligned}
& \chi_{j}(\cdot, t) \in C^{0}\left(\Gamma_{h}(t)\right), \quad \chi_{j}\left(X_{i}(t), t\right)=\delta_{i j}, \\
& \chi_{j}(\cdot, t) T_{k}, \text { is linear affine, }
\end{aligned}
$$

and on $T_{k} \in \mathcal{T}_{h}(t)$

$$
\chi_{j} e=\lambda_{k}, \quad \text { for each } e \in \mathcal{T}_{h}(t)
$$

where $k=k\left(T_{k}, j\right)$ and $\left(\lambda_{1}, \lambda_{2}, \lambda_{3}\right)$ are the barycentric coordinates. On $\Gamma_{h}(t)$ we define the discrete material velocity

$$
\boldsymbol{v}_{h}=\sum_{j=1}^{N} \dot{X}_{j}(t) \chi_{j}
$$

and the discrete material derivative

$$
\partial_{h}^{\bullet} \phi=\phi_{t}+v_{h} \cdot \nabla \phi
$$

Proposition 3.1 (Transport property). On $\Gamma_{h}(t)$, for each $j=1, \ldots, N$,

$$
\partial_{h}^{\bullet} \chi_{j}=0
$$

and for each $\phi=\sum_{j=1}^{N} \gamma_{j}(t) \chi_{j} \in S_{h}(t)$ then $\partial_{h}^{\bullet} \phi=$ $\sum_{j=1}^{N} \dot{\gamma}_{j}(t) \chi_{j}$.

Proof. See [32].

We seek approximations of $U(\cdot, t) \in S_{h}(t)$ to $u$ and $W(\cdot, t) \in$ $S_{h}(t)$ to $w$. Since $\left\{\chi_{j}(\cdot, t)\right\}_{j=1}^{N}$ is the basis of $S_{h}(t)$ we know that for each $U(\cdot, t) \in S_{h}(t)$, each $W(\cdot, t) \in S_{h}(t)$ and each $t \in$ $\left[0, t_{F}\right]$ there exist unique $\boldsymbol{\alpha}_{u}=\left\{\alpha_{u}^{1}(t), \ldots, \alpha_{u}^{N}(t)\right\}$ and $\boldsymbol{\alpha}_{w}=$ $\left\{\alpha_{w}^{1}(t), \ldots, \alpha_{w}^{N}(t)\right\}$ satisfying

$U(\cdot, t)=\sum_{j=1}^{N} \alpha_{u}^{j}(t) \chi_{j}(\cdot, t), \quad$ and $\quad W(\cdot, t)=\sum_{j=1}^{N} \alpha_{w}^{j}(t) \chi_{j}(\cdot, t)$. 
Substituting $U(\cdot, t), W(\cdot, t), \Gamma_{h}(t)$, and $\phi \in S_{h}(t)$ for $u, w, \Gamma(t)$, and $\varphi$ in (3.4) we obtain

$$
\begin{aligned}
& \frac{d}{d t} \int_{\Gamma_{h}(t)} \sum_{j=1}^{N} \alpha_{u, t}^{j} \chi_{j} \phi-\int_{\Gamma^{\prime}(t)} \sum_{j=1}^{N} \alpha_{u, t}^{j} \chi_{j} \partial_{h}^{\bullet} \phi \\
& \quad+\int_{\Gamma_{h}(t)} \sum_{j=1}^{N} \alpha_{u}^{j} \chi_{j} \phi \nabla_{\Gamma_{h}} \cdot \boldsymbol{v}_{h}+\int_{\Gamma_{h}(t)} \sum_{j=1}^{N} \alpha_{u}^{j}(t) \nabla_{\Gamma_{h}} \chi_{j} \cdot \nabla_{\Gamma_{h}(t)} \phi \\
& \quad+\int_{\Gamma_{h}(t)} \sum_{j=1}^{N} \alpha_{w}^{j}(t) \nabla_{\Gamma_{h}} \chi_{j} \cdot \nabla_{\Gamma_{h}(t)} \phi=\gamma \int_{\Gamma_{h}} f \phi
\end{aligned}
$$

for all $\phi \in S_{h}(t)$. Taking $\phi=\chi_{k}, k=1, \ldots, N$ and using the transport property of the basis functions we obtain

$$
\left\{\begin{array}{lll}
\frac{d}{d t}\left(\mathcal{M}(t) \boldsymbol{\alpha}_{u}\right)+\mathcal{S}(t) \boldsymbol{\alpha}_{u}+d_{w} \mathcal{S}(t) \boldsymbol{\alpha}_{w} & = & \gamma \boldsymbol{F}(t), \\
\frac{d}{d t}\left(\mathcal{M}(t) \boldsymbol{\alpha}_{w}\right)+d \mathcal{S}(t) \boldsymbol{\alpha}_{u}+d_{u} \mathcal{S}(t) \boldsymbol{\alpha}_{w} & = & \gamma \boldsymbol{G}(t),
\end{array}\right.
$$

where $\mathcal{M}(t)$ is the evolving mass matrix defined by

$$
\mathcal{M}(t)_{j k}=\int_{\Gamma_{h}(t)} \chi_{j} \chi_{k}
$$

$\mathcal{S}(t)$ is the evolving stiffness matrix defined by

$$
\mathcal{S}(t)_{j k}=\int_{\Gamma_{h}(t)} \nabla_{\Gamma_{h}} \chi_{j} \cdot \nabla_{\Gamma_{h}} \chi_{k},
$$

and $\boldsymbol{F}$ and $\boldsymbol{G}$ are the right-hand side defined by $\boldsymbol{F}_{i}=$ $\int_{\Gamma_{h}(t)} f(\boldsymbol{U}, \boldsymbol{W}) \chi_{i}$ and $\boldsymbol{G}_{i}=\int_{\Gamma_{h}(t)} g(\boldsymbol{U}, \boldsymbol{W}) \chi_{i}$, respectively.

\section{Time discretization}

We illustrate the time discretization of the reactiondiffusion system with activator-depleted reaction kinetics as follows. Other reaction kinetics can be treated in a similar fashion. For the time discretization, let $T_{m}$ denote the maximum time of interest, $\tau$ denote the time step, and $n_{T}$ be a fixed nonnegative integer, then $\tau=\frac{T_{m}}{n_{T}}$ and $t_{n}=n \tau$, $n=0,1,2, \ldots, n_{T}$. We denote the approximate solution at time $t_{n}$ by $U^{n}=U\left(\cdot, t_{n}\right)$, and $W^{n}=W\left(\cdot, t_{n}\right)$, thus $\left(U^{n}, W^{n}\right) \in S_{h}$ represents the solution at time $n \tau$ and $\Gamma_{h}^{n}=\Gamma_{h}(n \tau)$. Let $U^{0}$, $W^{0} \in S_{h}(0)$ be given. For $n=0, \ldots, n_{T}$ solve the nonlinear system

$$
\begin{gathered}
\frac{1}{\tau} \int_{\Gamma_{h}^{n+1}} U^{n+1} \chi_{j}^{n+1}+\int_{\Gamma_{h}^{n+1}} \nabla_{\Gamma_{h}^{n}} U^{n+1} \cdot \nabla_{\Gamma_{h}^{n}} \chi_{j}^{n+1}+d_{w} \int_{\Gamma_{h}^{n+1}} \nabla_{\Gamma_{h}^{n}} W^{n+1} \cdot \nabla_{\Gamma_{h}^{n}} \chi_{j}^{n+1}=\frac{1}{\tau} \int_{\Gamma_{h}^{n}} U^{n} \chi_{j}^{n}+\gamma \int_{\Gamma_{h}^{n+1}} f\left(U^{n+1}, W^{n+1}\right) \chi_{j}^{n+1}, \\
\frac{1}{\tau} \int_{\Gamma_{h}^{n+1}} W^{n+1} \chi_{j}^{n+1}+d \int_{\Gamma_{h}^{n+1}} \nabla_{\Gamma_{h}^{n}} W^{n+1} \cdot \nabla_{\Gamma_{h}^{n}} \chi_{j}^{n+1}+d_{u} \int_{\Gamma_{h}^{n+1}} \nabla_{\Gamma_{h}^{n}} U^{n+1} \cdot \nabla_{\Gamma_{h}^{n}} \chi_{j}^{n+1}=\frac{1}{\tau} \int_{\Gamma_{h}^{n}} W^{n} \chi_{j}^{n}+\gamma \int_{\Gamma_{h}^{n+1}} g\left(U^{n+1}, W^{n+1}\right) \chi_{j}^{n+1},
\end{gathered}
$$

for all $j=1, \ldots, N$. To linearize $f\left(U^{n+1}, W^{n+1}\right)$ and $g\left(U^{n+1}, W^{n+1}\right)$ we assume slow deformation of the evolving surface, which allows us to write $\left(U^{n+1}\right)^{2} \approx U^{n} U^{n+1}$ [46]. Using this linearization, we can derive the following fully discrete algorithm:

Let $U^{0}, W^{0} \in S_{h}(0)$ be given. For $n=0, \ldots, n_{T}$ solve the linear system

$$
\begin{aligned}
& \left(\frac{1}{\tau}+\gamma\right) \int_{\Gamma_{h}^{n+1}} U^{n+1} \chi_{j}^{n+1}+\int_{\Gamma_{h}^{n+1}} \nabla_{\Gamma_{h}^{n}} U^{n+1} \cdot \nabla_{\Gamma_{h}^{n}} \chi_{j}^{n+1}-\gamma \int_{\Gamma_{h}^{n+1}} U^{n} W^{n} U^{n+1} \chi_{j}^{n+1} \\
& \quad=\frac{1}{\tau} \int_{\Gamma^{n}} U^{n} \chi_{j}^{n}+d_{w} \int_{\Gamma_{h}^{n}} \nabla_{\Gamma_{h}^{n}} W^{n} \cdot \nabla_{\Gamma_{h}^{n}} \chi_{j}^{n}+\gamma a \int_{\Gamma_{h}^{n}} \chi_{j}^{n}, \\
& \frac{1}{\tau} \int_{\Gamma_{h}^{n+1}} W^{n+1} \chi_{j}^{n+1}+d \int_{\Gamma_{h}^{n+1}} \nabla_{\Gamma_{h}^{n}} W^{n+1} \cdot \nabla_{\Gamma_{h}^{n}} \chi_{j}^{n+1}+\gamma \int_{\Gamma_{h}^{n+1}}\left(U^{n+1}\right)^{2} W^{n+1} \chi_{j}^{n+1} \\
& \quad=\frac{1}{\tau} \int_{\Gamma^{n}} W^{n} \chi_{j}^{n}+d_{u} \int_{\Gamma_{h}^{n+1}} \nabla_{\Gamma_{h}^{n}} U^{n} \cdot \nabla_{\Gamma_{h}^{n}} \chi_{j}^{n}+\gamma b \int_{\Gamma_{h}^{n}} \chi_{j}^{n},
\end{aligned}
$$

for all $j=1, \ldots, N$. Using matrix representation we can write

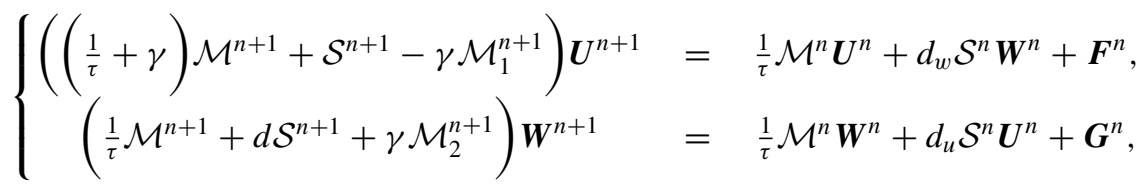

with

$$
\begin{gathered}
\mathcal{M}_{i j}^{n}=\int_{\Gamma_{h}^{n}} \chi_{i}^{n} \chi_{j}^{n}, \quad \mathcal{M}_{1 i j}^{n}=\int_{\Gamma_{h}^{n}} U^{n-1} W^{n-1} \chi_{i}^{n} \chi_{j}^{n}, \quad \mathcal{M}_{2 i j}^{n}=\int_{\Gamma_{h}^{n}}\left(U^{n+1}\right)^{2} \chi_{i}^{n} \chi_{j}^{n}, \\
\mathcal{S}_{i j}^{n}=\int_{\Gamma_{h}^{n}} \nabla_{\Gamma_{h}^{n}} \chi_{i}^{n} \cdot \nabla_{\Gamma_{h}^{n}} \chi_{j}^{n}, \quad \boldsymbol{F}^{n}{ }_{i}=\gamma a \int_{\Gamma_{h}^{n}} \chi_{i}^{n}, \quad \text { and } \quad \boldsymbol{G}^{n}{ }_{i}=\gamma b \int_{\Gamma_{h}^{n}} \chi_{i}^{n},
\end{gathered}
$$



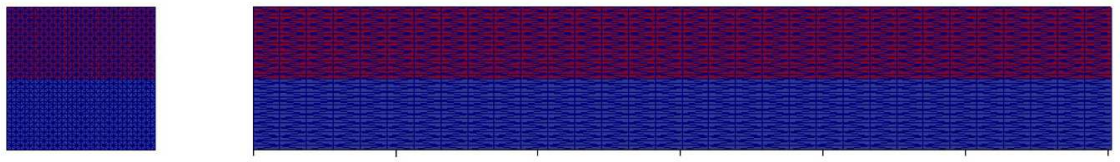

0.0

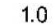

2.0

4.0

5.0

(a)
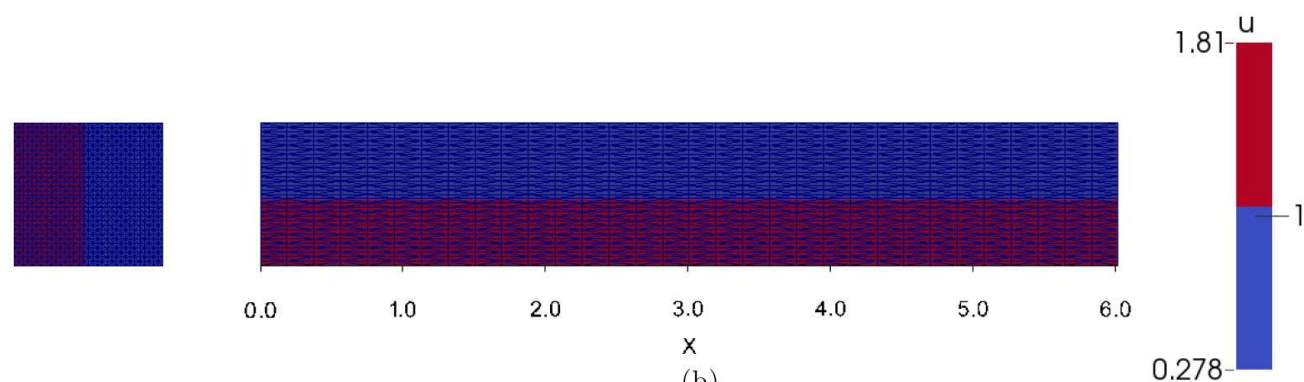

(b)

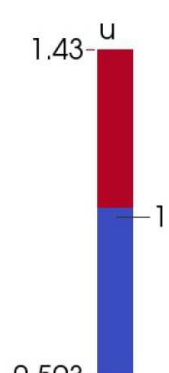

(c)

FIG. 1. (Color online) Numerical simulations corresponding to the chemical specie $u$ of the model equations (2.1) at $t=5$ (left column) and $t=180$ (right column). The $w$ simulations are omitted since these are out of phase to those of $u$. The initial square domain reaches approximately the final size [6,0] after growing according to the growth function defined by (4.4). In all these simulations we take the parameter values $d=10, a=0.1, b=0.9, \gamma=29$ and vary the cross-diffusion coefficients as (a) $d_{u}=1, d_{w}=1$, (b) $d_{u}=0, d_{w}=1$, and (c) $d_{u}=0$, $d_{w}=0$. We observe the formation of a stripe pattern as the domain evolves along the $x$ axis. The solution profiles with cross-diffusion in the $u$ equation only have the highest amplitude while those without cross diffusion have the lowest. This confirms our theoretical predictions published earlier in [25], which proved that cross-diffusion in the $u$ equation variable only induces the largest parameter space than either without cross-diffusion or with cross-diffusion in both equations.

where $\chi_{i}^{n} \in S_{h}^{n}=\left\{\chi \in C^{0}\left(\left(\Gamma_{h}(n \tau)\right)\right):\left.\chi\right|_{e}\right.$ is linear affine for each $e \in \mathcal{T}_{h}(n \tau)$. The resulting systems of linear algebraic equations are solved efficiently using the conjugate gradient method [47].

\section{NUMERICAL SIMULATIONS}

In all the simulations, initial conditions are taken as small random perturbations of the uniform steady state $\left(a+b, \frac{b}{(a+b)^{2}}\right)$, which is obtained by setting the kinetic functions to zero, i.e.,

$$
\left\{\begin{array}{l}
f(u, w)=a-u+u^{2} w=0, \\
g(u, w)=b-u^{2} w=0 .
\end{array}\right.
$$

As for boundary conditions, either they are not necessary if the domain is a surface of empty boundary or otherwise homogeneous Neumann boundary conditions are imposed. The implementation of the numerical schemes has been carried out using the finite element toolbox ALBERTA [48]. In all our simulations, threshold shading is applied as follows: we shade with blue (gray shading in black and white) if the values of the chemical species, say, $u>(a+b)$; otherwise, we shade with red (dark black shading in black and white). Since the solution profiles of $u$ are 180 degrees out of phase with those of $w$, we only plot contours corresponding to the chemical specie $u$; those of $w$ can be deduced from the $u$ patterns. A plausible biological justification is that chemical species above a certain threshold will express themselves differently (in the presence of chemical substances) from those under the threshold thereby generating spatial structure.

\section{A. Diffusion-induced pattern formation on evolving planar domains}

In this section we illustrate a computational framework in two dimensions that allows us to move the internal mesh given an evolution law of the boundary surface. The same approach could be used in three dimensions. To start the numerical simulation we must provide a conformal triangulation on the initial domain. The nodes on the boundary will evolve 

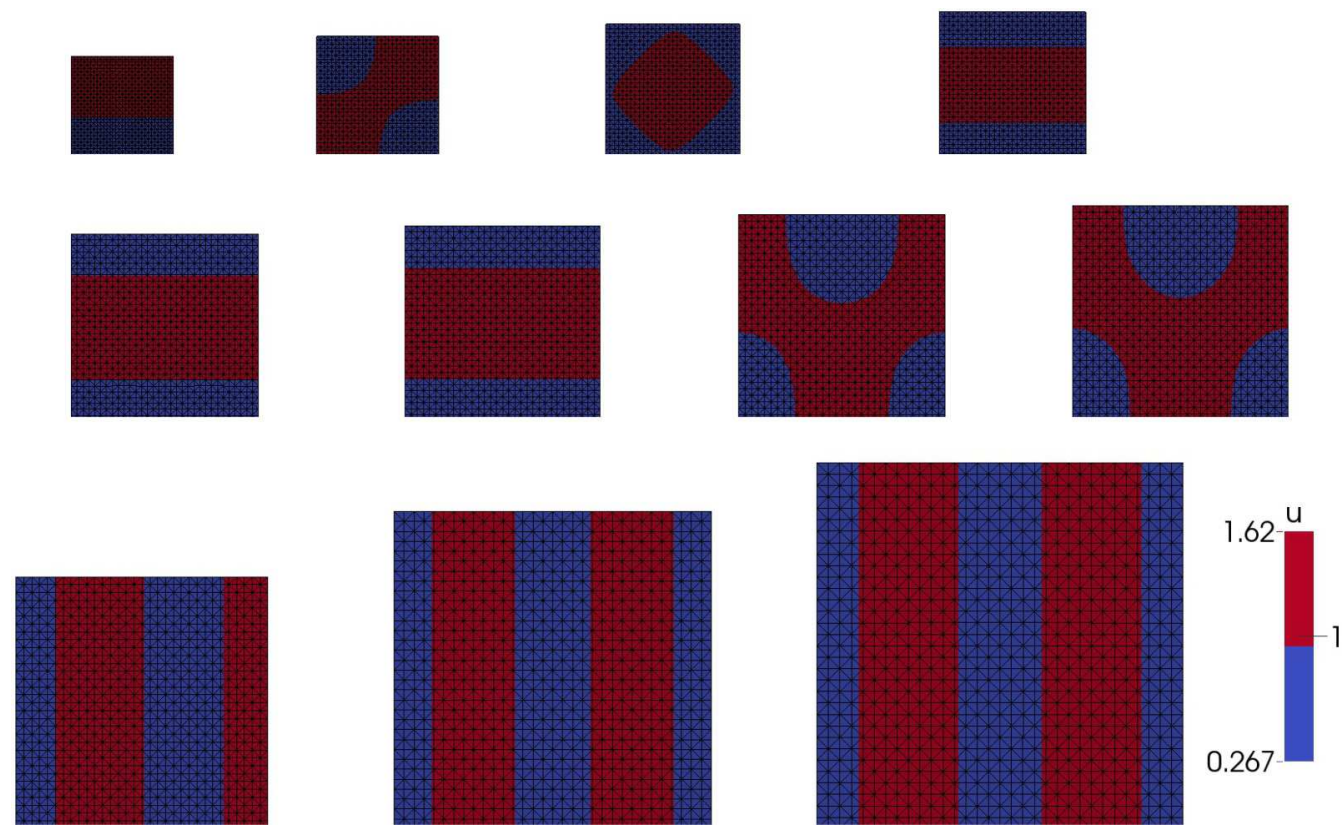

FIG. 2. (Color online) Numerical simulations corresponding to the chemical specie $u$ of the model equations (2.1) with model parameters $d=10, a=0.1, b=0.9, d_{u}=1, d_{w}=1$, and $\gamma=29$. The initial unit square domain grows exponentially to approximately 4.5 times its original size, growth is positive along the diagonal. Snapshots are shown at times $t=26,50,65,80,130,140,154,165,210,290$, and 350, respectively. The reaction-diffusion system with cross-diffusion in both the $u$ and $w$ equations exhibit a variety of transient patterns: stripes, spots, and circular patterns as the domain evolves. The bifurcation selection process seem to occur slightly faster than when cross-diffusion is present in only the $u$ equation (compare with Fig. 3).

according to a prescribed velocity, but the positions of the internal nodes must be calculated. We have used a common technique known as the (segment) spring analogy [49], which consists of replacing the mesh by fictitious springs. The equilibrium lengths of the springs are equal to the lengths of the edges in the initial triangulation. The force at each node $i$ is determined by

$$
\boldsymbol{F}_{i}=\sum_{i=1}^{n_{i}} k_{i j}\left(\delta_{i}-\delta_{j}\right),
$$

where $\delta_{i}$ is the displacement of node $i, n_{i}$ is the number of nodes surrounding node $i$, and $k_{i j}$ is the spring stiffness, which according to [50] for a given edge $i-j$, is of the form

$$
k_{i j}=\frac{1}{\sqrt{\left(x_{i}-x_{j}\right)^{2}+\left(y_{i}-y_{j}\right)^{2}}} .
$$

At static equilibrium, $\boldsymbol{F}_{i}$ is zero for all nodes and prescribing the displacements on the boundary and using nodal coordinates of the previous time step, we obtain a system of linear equations whose unknowns are the displacements $\delta_{i}$. After solving this system, the nodal coordinates are updated in the following manner:

$$
\boldsymbol{x}_{i}^{\text {new }}=\boldsymbol{x}_{i}^{\text {old }}+\delta_{i} .
$$

Remark 4.1. If the domain or surface evolution is known a priori, for example, in the form of

$$
\boldsymbol{x}(t)=\rho(t) \boldsymbol{x}(0),
$$

where $\boldsymbol{x}(0)$ is the initial domain or surface and $\rho(t)$ is the evolution law, then it is not necessary to use the spring analogy described above; instead the whole triangulation is simply evolved according to the evolution law prescribed.

\section{Evolving a unit square domain along one of the axis}

In this example $\Gamma(0)$ is the unit square in $\mathbb{R}^{2}$ which is evolving along the $x$ axis according to the following evolution law:

$$
(x(t), y(t))=\left(x(0) e^{\sigma t}, 0\right),
$$

where we assume the growth is slow, so that $\sigma$ is considered a small parameter.

Our first example is inspired by the simulation results shown in Figs. 2(a)-2(b) of [4]. To proceed, we will take the same kinetic parameter values, which are given by $a=0.1$ and $b=$ 0.9. These have been shown to belong to the classical Turing space in the absence of cross-diffusion [7]. We will take $\gamma=$ 29 and $d=10$ as outlined in [4]. We will assume that a unit square domain is evolving until it reaches approximately the final size $[6,0]$ along the $x$ axis only. Furthermore, we take the growth rate to be given by $\sigma=0.01$.

In Fig. 1 we present numerical solutions corresponding to the $u$ chemical species only; those of $w$ are out of phase. In Fig. 1(a) we compute solutions for the model system with cross-diffusion in both the $u$ and $w$ equations; in Fig. 1(b) cross-diffusion is only in the $u$ equation, and in Fig. 1(c) no cross-diffusion in either equation. We observe the formation of a single stripe described analytically by the $(1,0)$ mode. It can be shown analytically, in the absence of cross-diffusion, that the $(1,0)$ mode is always excitable when the unit square is evolving in a one-dimensional fashion along the $x$ axis. This theoretical result holds true when cross-diffusion is introduced. 

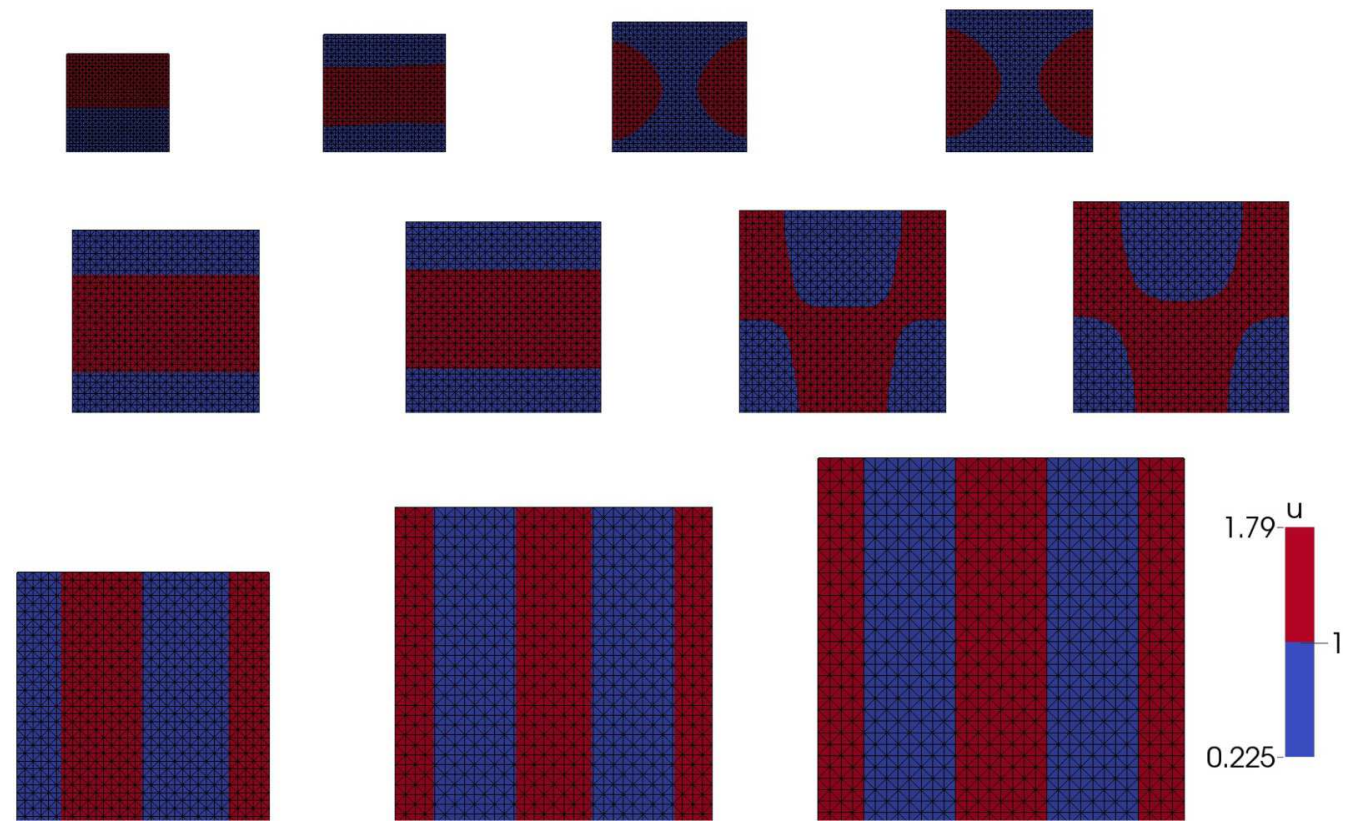

FIG. 3. (Color online) Numerical simulations corresponding to the chemical specie $u$ of the model equations (2.1) with parameters $d=10$, $a=0.1, b=0.9, d_{u}=0, d_{w}=1$, and $\gamma=29$. Snapshots are shown at times $t=26,50,65,80,130,140,154,165,210,290$, and 350, respectively. The reaction-diffusion system with cross-diffusion in the $u$ equations only exhibit a variety of transient patterns: stripes, spots, and circular patterns as the domain evolves. The bifurcation selection process of the transient patterns occurs slightly later than when cross-diffusion is present in both species (compare with Fig. 2).

The only difference between solutions with or without crossdiffusion is in their amplitude. Solution profiles with crossdiffusion in the $u$ equation have the highest amplitude. This confirms our most recent theoretical results, which proved that introducing cross-diffusion in the $u$ equation only induces (and much earlier) larger parameter spaces than those either with cross-diffusion in both equations or without cross-diffusion at all [25].
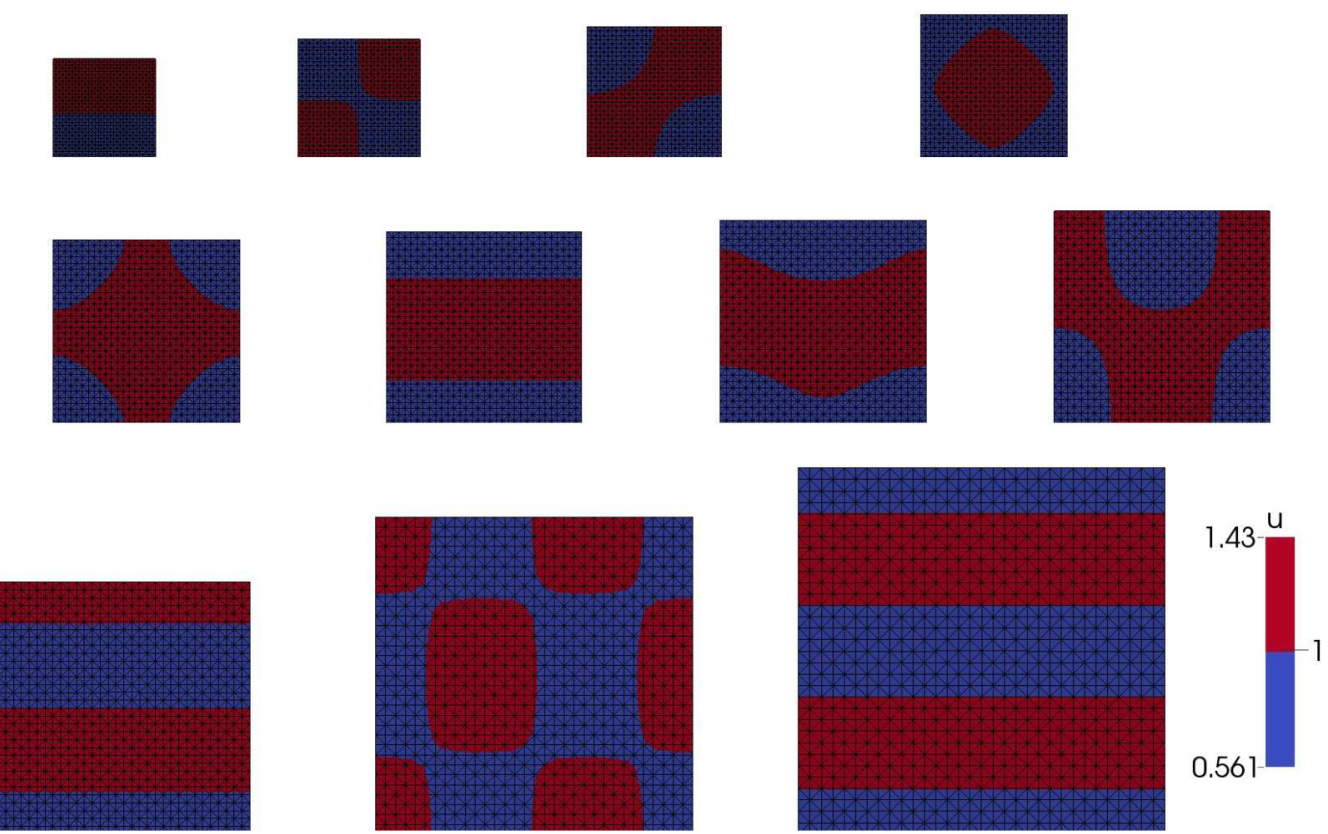

FIG. 4. (Color online) Numerical simulations corresponding to the chemical specie $u$ of the model equations (2.1) with parameters $d=10, a=0.1, b=0.9, d_{u}=0, d_{w}=0$, and $\gamma=29$. Snapshots are shown at times $t=26,50,65,80,130,140,154,165,210,290$, and 350, respectively. In the absence of cross-diffusion we observe patterns identical to those obtained in previous studies for the classical reaction-diffusion system posed on an evolving square domain [4]. We observe rapid transitions between patterns: stripes turning into spots, oblique and circular patterns. The regions of instability are small compared to those when cross-diffusion is present either in both species or in the $u$ equation only. 


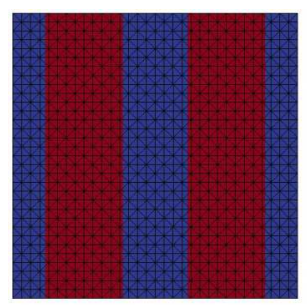

(a)

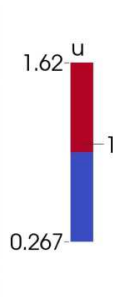

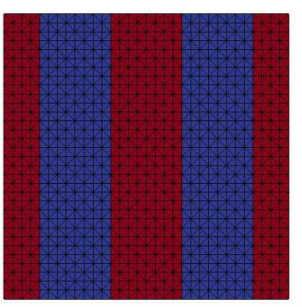

(b)

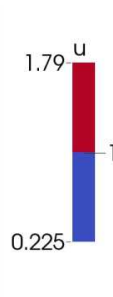

FIG. 5. (Color online) Transient patterns exhibited for the $u$ species corresponding to the model equations (2.1) at time $t=350$ for (a) $d_{u}=1$ and $d_{w}=1$ : the reaction-diffusion system with cross-diffusion in both equations, (b) $d_{u}=0$ and $d_{w}=1$ : the reaction-diffusion system with cross-diffusion in the $u$ equation only, and (c) $d_{u}=0$ and $d_{w}=0$ : the reaction-diffusion system without cross-diffusion in both equations. These patterns demonstrate that, for appropriate model parameter values, reaction-diffusion systems with or without cross-diffusion can select identical transient patterns during the bifurcation process, even though the transient process throughout the evolution process is different.

It must be observed that the transient pattern is independent of the initial random fluctuations around the uniform state. Transient patterns emerging soon after growth show two types of horizontal and vertical stripes (left column of Fig. 1). If the model equations are solved on a stationary domain (in this case the unit square) in the absence of domain growth, then pattern selection is critically dependent on the initial conditions. To this end, random initial fluctuations around the uniform state will act as a basin of attraction; the pattern to be converged to depends on how close it is to the initial conditions. Pattern sensitivity to initial conditions on static domains and surfaces has been a major criticism of Turing's theory for pattern formation [7,8]. By introduction domain growth, pattern transition during growth development is independent of the initial random fluctuations around the uniform state. The horizontal stripe pattern that persists during domain growth is unstable when domain growth is switched off; instead vertical stripes representing excitable modes of the form $(m, 0)$, with $m>1$ are obtained (see [4] for examples in the absence of cross-diffusion).

\section{Evolving a unit square domain along the diagonal}

In our second example, all model parameter and diffusion coefficients remain fixed as those in the example above. The only change is the evolution of the square domain under the exponential growth. Here we want to explore if cross-diffusion does induce different transient patterns during domain evolution when the unit square domain evolves uniformly isotropically, along the diagonal of the first positive quadrant. Again, we compare our results to those shown in
Fig. 4 of [4], which are reproduced exactly using our numerical method (compare Fig. 4 with Fig. 4 in [4]). Figures 2-4 exhibit patterns obtained with

(1) Cross-diffusion in both the $u$ and $w$ equations (Fig. 2)

(2) Cross-diffusion in the $u$ equation only (Fig. 3)

(3) Without cross-diffusion (Fig. 4).

In all our figures, at time $t=26$ we observe the formation of stripe patterns exhibiting the $(1,0)$ mode as described in Fig. 1. As the domain continues to evolve, different transient patterns are observed for the three different cases. For example, at time $t=50$, the bifurcation process for reaction-diffusion system with cross-diffusion in both components and without cross-diffusion occurs much faster than that of the system with cross-diffusion in the $u$ equation only. At time $t=80$, we observe a stripe pattern for the reaction-diffusion system with cross-diffusion in both equations, while spot patterns form for the system with cross-diffusion in the $u$ equation only. The system without cross-diffusion exhibits the formation of a single spot. The bifurcation sequence for all models also selects the same excitable wave numbers (and therefore exhibits identical transient patterns) during growth development. In Fig. 5 we observe, for all model systems, identical patterns (except for symmetry) at the final time $t=350$. These results clearly demonstrate the effects of cross-diffusion to the formation of pattern formation.

\section{B. Cross-diffusion induced pattern formation on evolving domains}

In this section, we start to depart from classical Turing parameter values and consider parameter values $a$ and $b$ that
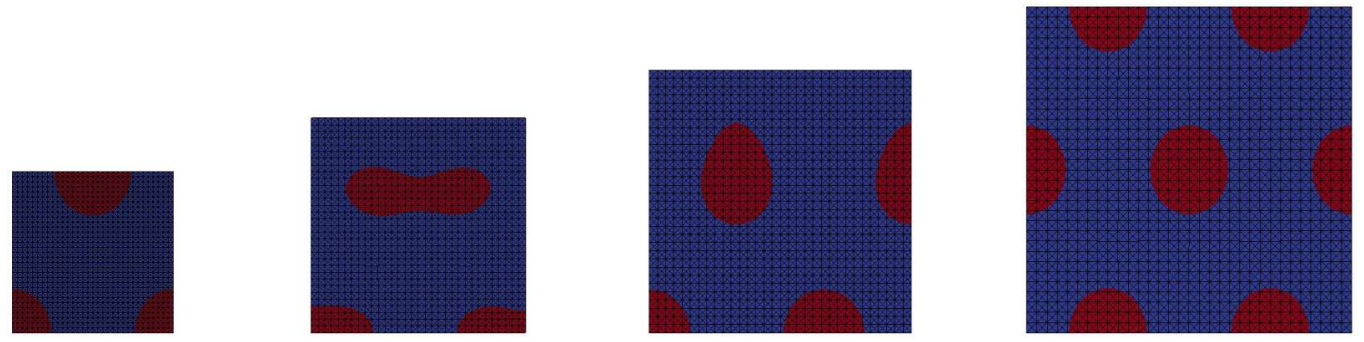

FIG. 6. (Color online) Numerical simulations corresponding to the chemical specie $u$ of the model equations (2.1) with cross-diffusion in the $u$ equation only. We take parameters $d=1, a=0.1, b=0.1, d_{u}=0, d_{w}=0.5$, and $\gamma=200$. Snapshots are shown at times $t=5,40,71$, and 112 , respectively. We observe a continuous evolution of spot transient patterns as the domain continues to grow. 

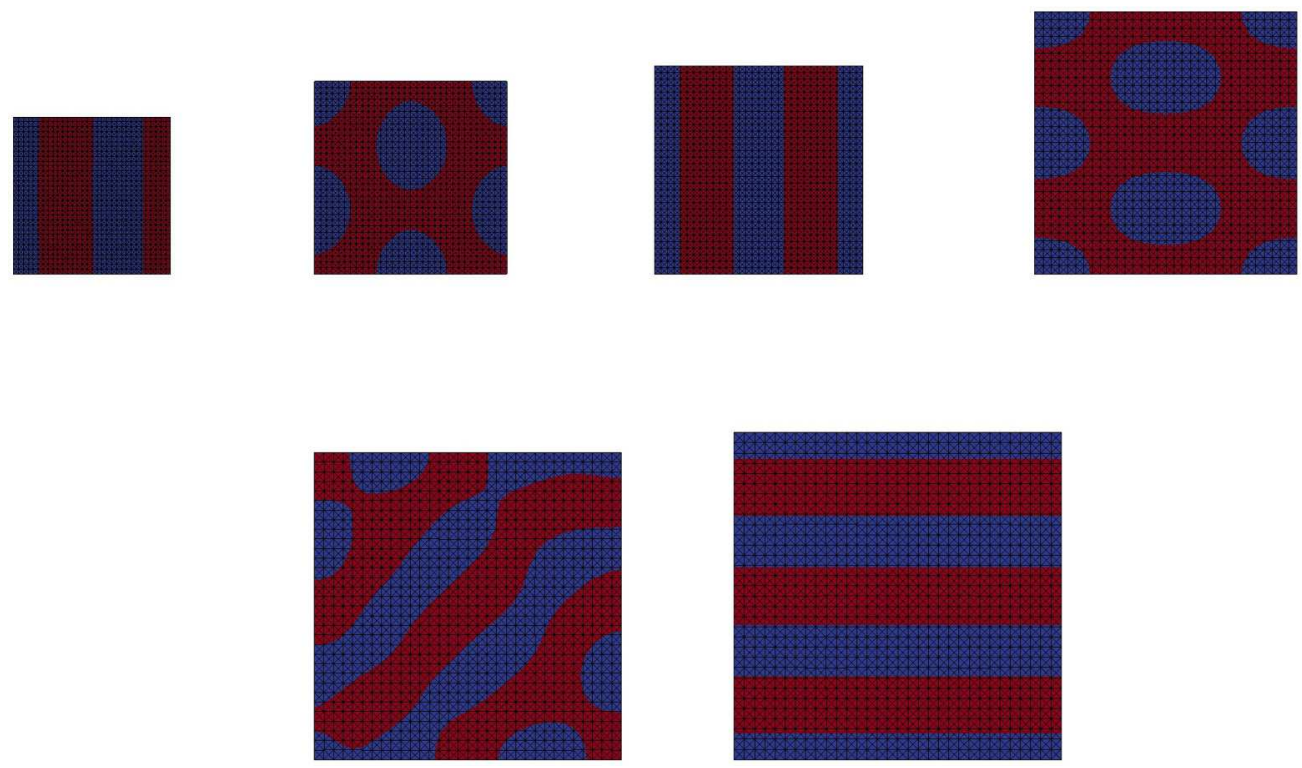

FIG. 7. (Color online) Numerical simulations corresponding to the chemical specie $u$ of the model equations (2.1) with cross-diffusion in the $u$ equation only. We take parameters $d=1, a=0.17, b=0.25, d_{u}=0, d_{w}=0.8$, and $\gamma=200$. Snapshots are shown at times $t=2,25$, $35,70,99$, and 112, respectively. We observe stripes evolving into spots, which further transient into stripes and spots with continuous growth. The spot patterns evolve into oblique patterns and these form stripes as the square reaches approximately twice its original size.

are selected from cross-diffusion induced parameter spaces. The parameter values are picked from parameter spaces published in Madzvamuse et al. [25]. For these parameter values, the reaction-diffusion system (2.1) gives rise to patterns only in the presence of cross-diffusion, without cross-diffusion no patterns form. To demonstrate the effects of cross-diffusion, we will contrast patterns formed in the presence of crossdiffusion to those obtained in the absence of cross-diffusion. In all our simulations in this section, $\Gamma(0)$ is the unit square and is evolving exponentially, uniformly, and isotropically, along the diagonal with growth rate $\sigma=0.01$. In all our simulations from here onwards, we plot snap shots of the domain and surface evolution without axis labels, and we plot these according to their relative physical scales.

\section{Cross-diffusion induced patterns on planar domains}

Unlike the case of standard reaction-diffusion systems, we assume that the activator $u$ and the inhibitor $w$ diffuse at equal rates (i.e., $d=1$ ). Furthermore, we will select the model parameter values $a=0.1$ and $b=0.1$ outside the classical Turing parameters; these belong to the cross-diffusion induced parameter space as shown in [25], Fig. 9(a). In order to induce patterning, we take $d_{u}=0, d_{w}=0.5$; i.e., we consider the reaction-diffusion system with cross-diffusion in the $u$ equation only. Figure 6 illustrates the formation of spot patterns as the unit square domain grows to approximately twice its original size. The observed patterns are formed only in the presence of cross-diffusion.

A second example involves two results as shown in Figs. 7 and 8 where we have selected the following parameter values: $d=1, a=0.17, b=0.25, d_{u}=0, d_{w}=0.8$ (Fig. 7) and $d=1, a=0.18, b=0.2, d_{u}=1, d_{w}=0.7$ (Fig. 8). The model kinetic parameter values are chosen from the cross-diffusion-induced parameter space shown in [25],
Fig. 11(c). In Fig. 7 we observe the formation of spots and stripe patterns as the unit square domain evolves to approximately twice its original size. Oblique and spot patterns are exhibited at time $t=99$. Here cross-diffusion has been introduced only in the $u$ equation.
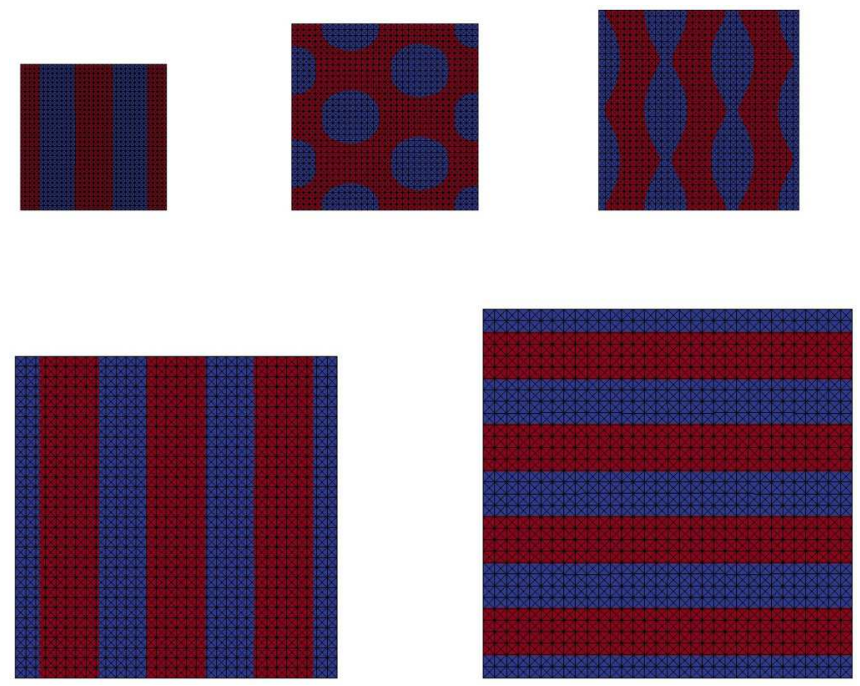

FIG. 8. (Color online) Numerical simulations corresponding to the chemical specie $u$ of the model equations (2.1) with crossdiffusion in both the $u$ and $w$ equations. We take parameters $d=1$, $a=0.18, b=0.2, d_{u}=1, d_{w}=0.7$, and $\gamma=200$. Snapshots are shown at times $t=5,34,44,85$, and 112, respectively. Again, we observe the formation of spots, stripes and zigzag patterns. The formation of these patterns seem to occur slightly later than those shown in Fig. 7. This could be a result of different parameter values or simply that the system with cross-diffusion in the $u$ equation induces patterning faster than the system when cross-diffusion is in both species. 
Introducing cross-diffusion in both species we observe the emergence of different transient patterns, stripes evolving into spots which in turn evolve into stripes. This could be a result of different parameter values or simply that the system with cross-diffusion in the $u$ equation induces patterning faster than the system when cross-diffusion is in both species. The most likely scenario is that the reaction-diffusion system with crossdiffusion in both components exhibits patterning much faster than when cross-diffusion is introduced in one component or without cross diffusion. For theoretical and computational evidence see [25]. It must be noted that the results presented in Figs. 7 and 8 are two different systems, with different model parameters. These will also influence the patterning process.

\section{Pattern formation on evolving surfaces}

For the first time, we present new results on pattern formation of the reaction-diffusion system with cross-diffusion on evolving surfaces. We will compare and contrast where possible patterns exhibited in the presence of cross-diffusion and those obtained in the absence of cross-diffusion. Furthermore, we will showcase cross-induced diffusion patterns on surfaces; parameter values are selected such that in the absence of cross-diffusion no patterns form.

\section{Evolving a unit sphere surface according to the logistic growth function}

Next, we consider the evolution of a unit sphere for which each material point $X(t)$ on $\Gamma(t)$ is evolving according to the logistic law

$$
X(t)=\rho(t) \boldsymbol{v},
$$

where $\rho(t)$ is a logistic growth function given by

$$
\rho(t)=\frac{e^{r t}}{1+\frac{1}{K}\left(e^{r t}-1\right)}, \quad r>0, \quad \text { and } \quad K>1 .
$$

In the above $K$ is the limiting final size of the evolving spherical surface. For illustrative purposes we take $K=1.5$ and $r=0.01$ in our experiment.

Figure 9 illustrates the evolution of the spherical surface driven by the logistic growth function. We observe the formation of spot patterns which are ellipsoidal in shape. Here, cross-diffusion is present only in the $u$ equation. We have taken the following parameter values $d=10, a=0.2$, $b=0.5, d_{u}=0, d_{w}=1$, and $\gamma=200$. Again, $a$ and $b$ are selected outside the classical Turing parameter space; these belong to the cross-diffusion parameter space.
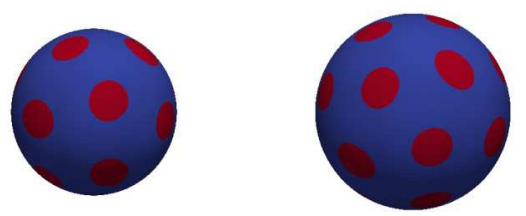

\section{Evolving a cylindrical surface according to an exponential growth}

Let us consider the evolution of a cylinder of radius 1 . For illustrative purposes we assume that the surface is evolving in the normal direction according to the following evolution law:

$$
X(t)=\rho(t) v X(0),
$$

where $\rho(t)=e^{r t}$, with $r=0.01$ and $\boldsymbol{v}$ is the unit normal to the surface. $X(0)$ is the initial surface at time $t=0$. Here we are simply expanding the surface uniformly isotropically in the normal direction.

We present two sets of results as shown in Fig. 10. In both cases, we plot numerical solutions corresponding to the $u$-chemical specie of the model equations with cross-diffusion in both the $u$ and $w$ equations with diffusion parameter values $d=10, d_{u}=1, d_{w}=1$, and we take $\gamma=200$. In the left column we fix $a=0.1$ and $b=0.9$; these belong to the classical Turing parameter space in the absence of cross-diffusion. In the right column, we fix $a=0.2$ and $b=0.5$; these belong outside the classical Turing parameter space, they belong to the cross-diffusion induced parameter space (see Fig. 3 in [25]).

The reaction-diffusion system without cross-diffusion induces mainly oblique stripe patterns around the cylindrical surface as the surface evolves. Large spot patterns are observed to form at later stages of the growth development. In the presence of cross-diffusion, we observe the formation of spot patterns with much smaller radii compared to those in the absence of cross-diffusion. In some cases, the spot patterns are of variable sizes.

\section{Anisotropic surface evolution: The effects of cross-diffusion}

In most biological systems involving growth development, surface evolution is anisotropic in many cases. To illustrate how cross-diffusion induces different patterns to those obtained from classical Turing patterns, we consider for illustrative purposes an anisotropic surface evolution where we define the initial surface by $\Gamma(0)$ and is given by

$$
25\left(x^{2}+y^{2}\right)-6 z^{2}=1, \quad-2 \leqslant z \leqslant 2 .
$$

We introduce anisotropic surface evolution by prescribing the surface evolution of the form

$$
\left\{\begin{array}{l}
x(t)=x(0)\left(1+r t\left\{1-0.2\left[z(0)^{2}+1\right]\right\},\right. \\
y(t)=y(0)\left(1+r t\left\{1-0.2\left[z(0)^{2}+1\right]\right\},\right. \\
z(t)=z(0)
\end{array}\right.
$$

where we take $r=0.01$.
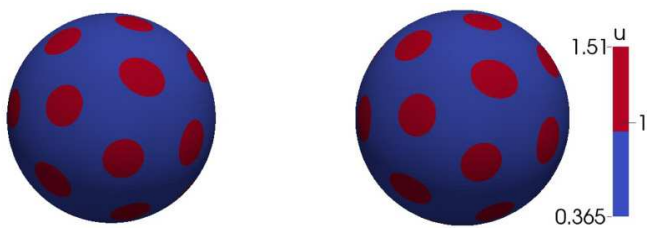

FIG. 9. (Color online) Cross-diffusion induced spot patterning on an evolving spherical surface whose evolution is defined by the logistic law (4.5). Snapshots of patterns corresponding to the chemical specie $u$ solving the model equation (2.1) with cross-diffusion in the $u$ equation only are shown at times $t=50,150,250$, and 350, respectively. Parameter values are taken as $d=10, a=0.2, b=0.5, d_{u}=0, d_{w}=1$, and $\gamma=200$. 

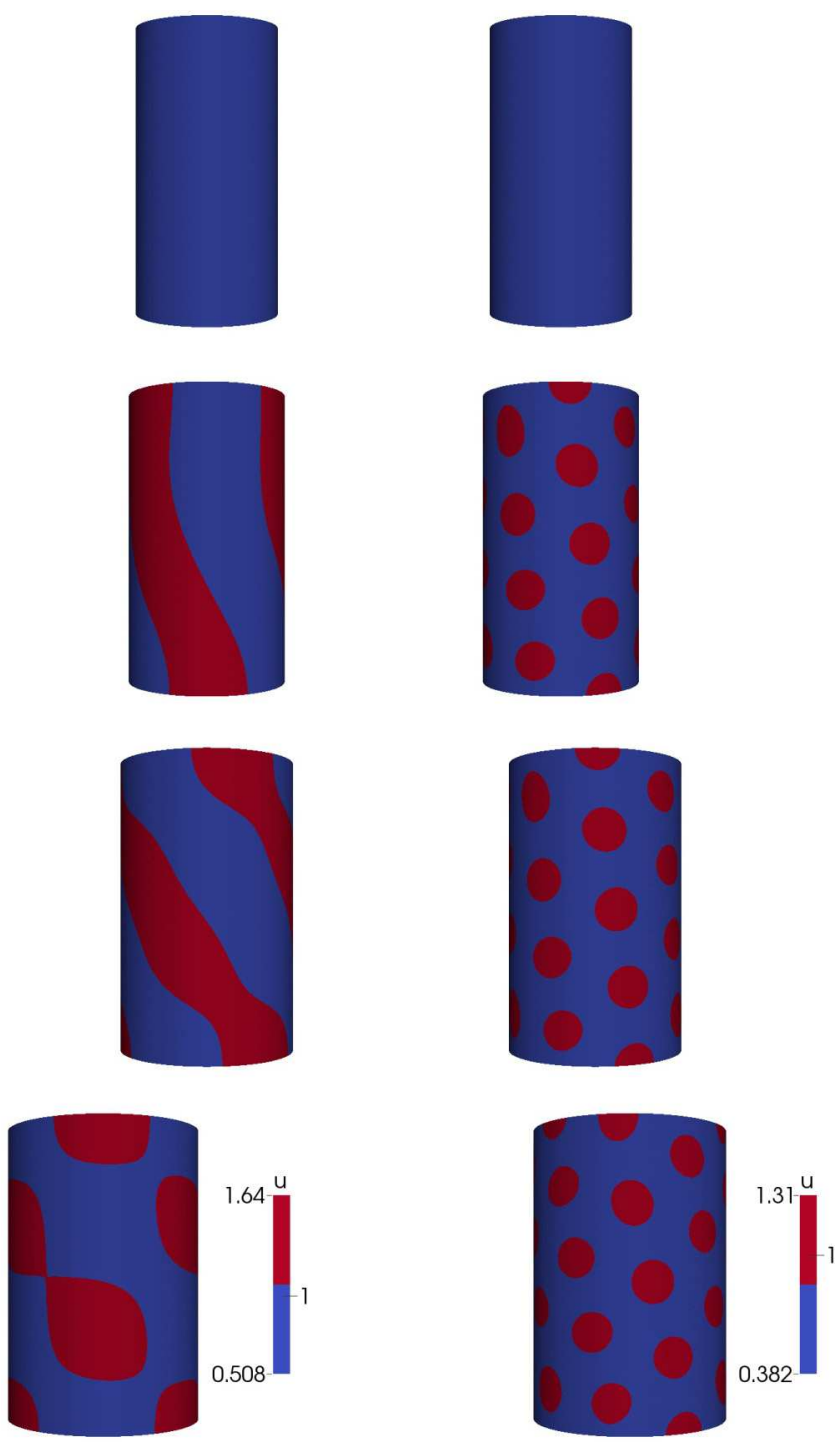

FIG. 10. (Color online) Numerical simulations corresponding to the chemical specie $u$ of the model equations (2.1) on an evolving cylindrical surface whose evolution is defined by the exponential growth (4.7). Parameter values are $d=10, d_{u}=1, d_{w}=1, \gamma=200$ with $a=0.1, b=0.9$ (left column), and $a=0.2, b=0.5$ (right column). Snapshots are shown at times $t=0,10,20$, and 30, respectively.

Figure 11 exhibits both the anisotropic surface evolution as well as the pattern formation process during growth development. The left column shows the formation of stripe-circular patterns corresponding to the chemical specie $u$ solving the model equations (2.1) in the absence of cross-diffusion. The right column shows the formation of spot patterns when cross-diffusion is present in both the $u$ and $w$ equations. Cross-diffusion induces substantially different, predominantly spot patterns as the surface continues to evolve. The spot patterns have a much smaller wavelength than those obtained when cross-diffusion is absent in the reaction-diffusion system. We observe that the model parameter values selected in the absence of cross-diffusion seem to induce the formation of stripe patterns as opposed to spot patterns.
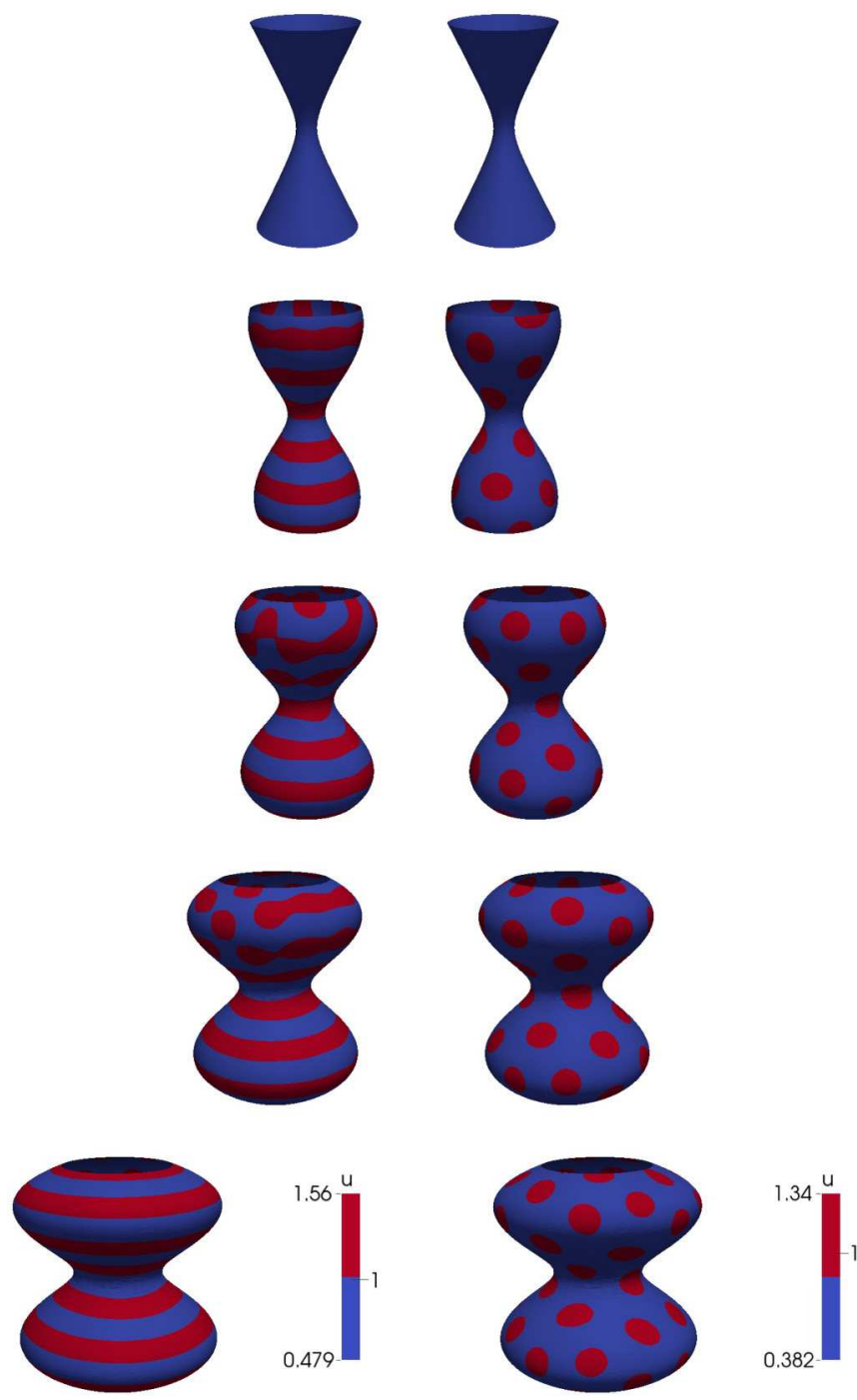

FIG. 11. (Color online) Patterns exhibited during an anisotropic surface evolution as defined by (4.9) at times $t=0,100,200,300$, and 400. Parameters are taken as $d=10, d_{u}=1, d_{w}=1, \gamma=200$, and $a=0.1, b=0.9$ (left column) and $a=0.2, b=0.5$ (right column).

\section{Concentration-driven surface evolution}

Our final example is again inspired by biological observations which support that in many processes where surface growth is involved, growth is driven partly by chemical species resident on the cell-membrane surface An example is that of cell polarization in cell biology due to responses to external signals through the outer cell membrane [51,52]. To model such concentration-driven surface evolution, we assume that a spherical surface is evolving according to the following evolution law:

$$
V=\delta u-\epsilon H,
$$

where $V$ is the normal velocity, $\delta$ is a growth rate, $H$ is the mean curvature of the surface, and $\epsilon$ is a small parameter meant to regularize the evolution law. We are using the definition of mean curvature to be the sum of the two principle curvatures. The details on how this velocity is implemented can be found in [31]. 


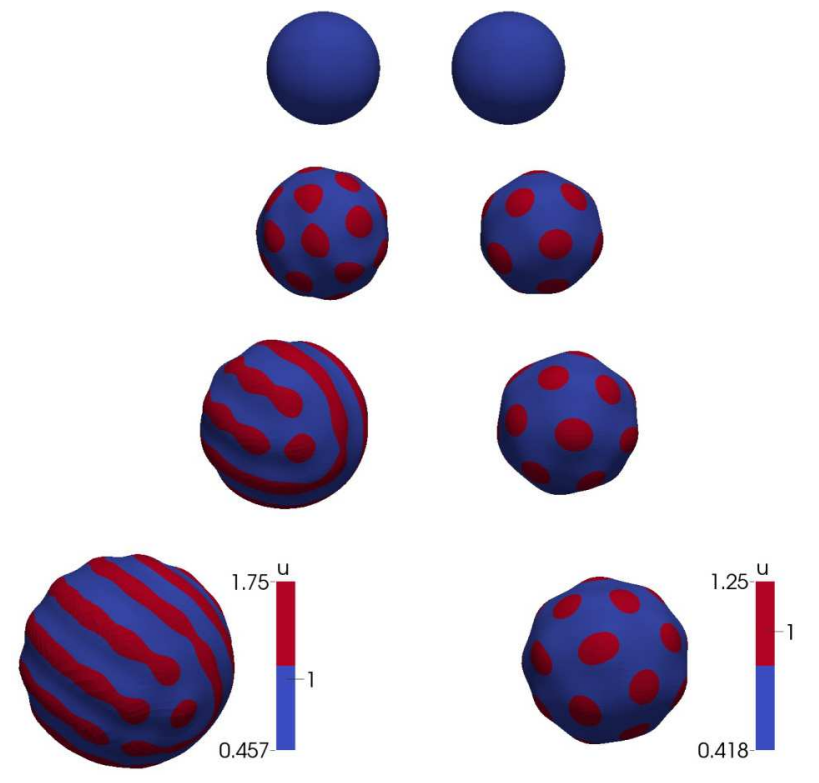

FIG. 12. (Color online) Pattern formation during the concentration-driven surface evolution according to the evolution law (4.10). Parameter values are taken as $d=10, d_{u}=1, d_{w}=1$, $\gamma=200$ with $a=0.1, b=0.9$ (left column), and $a=0.2, b=0.5$ (right column). Snapshots are shown at times $t=0,6,15$, and 25, respectively. Spots and stripe-circular patterns form when standard model parameter values are used (left column) while spot patterns are observed to form when model parameter values are selected from the cross-diffusion parameter space only. Note that the spheroidal surfaces in the left column are substantially larger than those in the right column due to the fact that large concentrations values of $u$ are obtained and these contribute to large surface evolution since the evolution is driven by the $u$ concentrations.

For illustrative purposes let us take parameter values $d=$ $10, d_{u}=1, d_{w}=1.0, \gamma=200$ and consider two sets of model values for $a$ and $b$, namely, $a=0.1, b=0.9$ and $a=0.2$, $b=0.5$. The latter set belongs to the cross-diffusion parameter space only. These will not give rise to patterning in the absence of cross-diffusion.

Figure 12 displays snapshots exhibiting patterns forming during surface evolution. The surface is evolving arbitrarily, driven by chemical species resident on the surface. Similarly to previous results, classical parameter values seem to favor the formation of stripe-circular patterns during growth development, while those induced by cross-diffusion exhibit the formation of spot patterns.

\section{CONCLUSION AND DISCUSSION}

This study has unraveled the effects of cross-diffusion to the theory of pattern formation on evolving domains and surfaces. These results present detailed comparisons between patterns formed with model parameter values selected such that they belong to the classical Turing parameter space and model parameter values selected such that they belong only to the parameter space induced by cross-diffusion. Our most revealing findings are the following:
(1) For the parameter values selected from the crossdiffusion-induced parameter spaces, spot patterns with smaller wave lengths form during domain and surface evolution.

(2) Classical model parameter values $a=0.1$ and $b=0.9$ as used currently in the literature seem to favor the formation of stripe or circular patterns (for the model parameter values we have considered).

(3) The reaction-diffusion system with cross-diffusion in the $u$ equation or in both the $u$ and $w$ equations induce pattern formation earlier than the case without cross-diffusion. This result supports our previous theoretical results proving that cross-diffusion induces instability faster than the classical reaction-diffusion system in the absence of cross-diffusion [25].

(4) As a result, pattern bifurcation transitions during growth development occur substantially different for the three model systems. A theoretical analytical framework on the bifurcation analysis of reaction-diffusion systems with or without cross-diffusion on evolving domains and surfaces is still largely missing in the current literature. Such a framework could help us understand the role of curvature, for example, on pattern formation during growth development. It is through the use of novel numerical methods such as the finite elements and its extensions that we are able to compute pattern evolution on realistic biological surfaces. These results reveal interesting bifurcation transitions that are yet to be proved rigorously.

Our findings have been made possible by the use and application of the evolving surface finite element method for solving highly nonlinear reaction-diffusion systems with cross-diffusion on complex evolving domains and surfaces [31-33]. The generality and applicability of our numerical method allows to study not only uniform isotropic and anisotropic surface evolution laws, but more complex geometric and concentration-driven evolution laws. In particular, the methodology handles quite easily such laws which are defined as geometric partial differential equations. In particular we have presented results for the cases when the domains and surfaces are evolving according to prescribed uniform isotropic and anisotropic evolution laws as well as the case when the surface description is an unknown quantity which must be determined as part of the problem.

Understanding the effects of cross-diffusion to the theory of pattern formation is crucial in many areas of research such as nanoparticles, surfactants, and polymers. Numerical methods such as those proposed in this article are critical to revealing pattern formation during growth development of biological systems. Our framework offers modelers, computational biologists, and numerical analysts with robust, efficient, and stable computational algorithms for studying such complex partial differential equations of reaction-cross-diffusion type on evolving domains and surfaces. In future work, we will focus on the stability and analysis of such models as well as developing bulk-surface methodologies that will allow us to couple surface dynamics to internal biochemical and biomechanical properties inside the surface.

\section{ACKNOWLEDGMENTS}

This work (A.M.) is supported by the following grants: an Engineering and Physical Sciences Research Council 
(EP/J016780/1) and the Leverhulme Trust Research Project Grant (RPG-2014-149). R.B. is supported by an EPSRC Grant (EP/J016780/1) as a Visiting Scholar to collaborate with A.M.
R.B. used the computational resources of the Center for Mathematics and Fundamental Applications of the University of Lisbon.
[1] E. J. Crampin, W. W. Hackborn, and P. K. Maini, Bull. Math. Biol. 64, 747 (2002).

[2] G. Hetzer, A. Madzvamuse, and W. Shen, Disc. Con. Dyn. Sys. 32, 3975 (2012).

[3] A. Madzvamuse, R. K. Thomas, P. K. Maini, and A. J. Wathen, Bull. Math. Biol. 64, 501 (2002).

[4] A. Madzvamuse, P. K. Maini, and A. J. Wathen, J. Comput. Phys. 190, 478 (2003).

[5] A. Madzvamuse, E. A. Gaffney, and P. K. Maini, J. Math. Biol. 61, 133 (2010).

[6] A. Turing, Phil. Trans. Royal Soc. B. 237, 37 (1952).

[7] J. D. Murray, Mathematical Biology. II, 3rd ed., Interdisciplinary Applied Mathematics Vol. 18 (Springer-Verlag, New York, 2003).

[8] J. Bard and I. Lauder, J. Theor. Bio. 45, 501 (1974).

[9] A. Gierer and H. Meinhardt, Kybernetik 12, 30 (1972).

[10] V. K. Vanag and I. R. Epstein, Phys. Chem. Chem. Phys. 11, 897 (2009).

[11] M. S. McAfree and O. Annunziata, Fluid Phase Equilibr. 356, 46 (2013).

[12] A. Vergara, F. Capuano, L. Paduano, and R. Sartorio, Macromolecules 39, 4500 (2006).

[13] V. Capasso and D. Liddo, J. Math. Biol. 32, 453 (1994).

[14] V. Capasso and D. Liddo, J. Math. Anal. Appl. 177, 510 (1993).

[15] G. Gambino, M. C. Lombardo, and M. Sammartino, Maths. Comp. Sim. 82, 1112 (2012).

[16] G. Gambino, M. C. Lombardo, and M. Sammartino, Nonlinear Anal. Real World Appl. 14, 1755 (2013).

[17] M. Iida and M. Mimura, J. Math. Biol. 53, 617 (2006).

[18] S. Kovács, Nonlin. Anal. 59, 567 (2004).

[19] F. Rossi, V. K. Vanag, E. Tiezzi, and I. R. Epstein, J. Phys. Chem. B 114, 8140 (2010).

[20] R. Ruiz-Baier and C. Tian, Nonlin. Anal. Real World Appl. 14, 601 (2013).

[21] C. Tian, Z. Lin, and M. Pedersen, Nonlin. Anal. Real World Appl. 11, 1036 (2010).

[22] Z. Xie, J. Math. Anal. Appl. 388, 539 (2012).

[23] J.-F. Zhang, W.-T. Li, and Y.-X. Wang, Nonlin. Anal. 74, 847 (2011).

[24] E. P. Zemskov, V. K. Vanag, and I. R. Epstein, Phys. Rev. E 84, 036216 (2011).

[25] A. Madzvamuse, H. S. Ndakwo, and R. Barreira, J. Math. Biol., doi:10.1007/s00285-014-0779-6.

[26] O. Lakkis, A. Madzvamuse, and C. Venkataraman, SIAM JNA 51, 2309 (2013).

[27] C. B. Macdonald, C. B. B. Merriman, and S. J. Ruuth, Proc. Natl. Acad. Sci. USA 110, 9209 (2013).
[28] C. B. Macdonald and S. J. Ruuth, SIAM J. Sci. Comput. 31, 4330 (2010).

[29] P. K. Maini, E. J. Crampin, A. Madzvamuse, A. J. Wathen, and R. D. K. Thomas, in Proceedings of the 5th European Conference for Mathematics and Theoretical Biology, Milan, Italy, edited by V. Capaso (Società Editrice Esculapio, Milan, Italy, 2002).

[30] M. A. J. Chaplain, M. Ganesh, and I. G. Graham, J. Math. Biol. 42, 387 (2001).

[31] R. Barreira, C. M. Elliott, and A. Madzvamuse, J. Math. Biol. 63, 1095 (2011).

[32] G. Dziuk and C. M. Elliott, J. Comp. Math. 25, 385 (2007).

[33] G. Dziuk and C. M. Elliott, Acta Numer. 22, 289 (2013).

[34] G. Dziuk and C. M. Elliott, Interfaces Free Bound. 10, 119 (2008).

[35] G. Dziuk and C. M. Elliott, Comput. Vis. Sci. 13, 17 (2010).

[36] A. Schmidt and K. G. Siebert, Level Set Methods and Dynamic Implicit Surfaces, Applied Mathematical Sciences Vol. 153 (Springer-Verlag, New York, 2003).

[37] J. A. Sethian, Level Set Methods and Fast Marching Methods, 2nd ed., Cambridge Monographs on Applied and Computational Mathematics (Cambridge University Press, Cambridge, 1999).

[38] K. Deckelnick, G. Dziuk, and C. M. Elliott, Acta Numer. 14, 139 (2005).

[39] C. M. Elliott, B. Stinner, V. Styles, and R. Welford, IMA J. Numer. Anal. 31, 786 (2011).

[40] D. A. Calhoun and C. Helzel, SIAM J. Sci. Comput. 31, 4066 (2010).

[41] J. B. Greer, A. L. Bertozzi, and G. Sapiro, J. Comput. Phys. 216, 216 (2006).

[42] S. E. Hieber and P. Koumoutsakos, J. Comput. Phys. 210, 342 (2005).

[43] I. Prigogine and R. Lefever, J. Chem. Phys. 48, 1695 (1968).

[44] J. Schnakenberg, J. Theor. Biol. 81, 389 (1979).

[45] A. Madzvamuse, A. J. Wathen, and P. K. Maini, J. Sci. Comp. 24, 247 (2005)

[46] A. Madzvamuse, J. Comp. Phys. 214, 239 (2006).

[47] Y. Saad, Iterative Methods for Sparse Linear Systems, SIAM, 2nd ed. (PWS Publishing Co., Philadelphia PA, 2003).

[48] A. Schmidt and K. G. Siebert, Design of Adaptive Element Software: The Finite Element Toolbox ALBERTA, Lecture Notes in Computational Science and Engineering Vol. 42 (Springer, Berlin, Heidelberg, New York, 2005).

[49] F. J. Blom, Int. J. Numer. Meth. Fluids 32, 647 (2000).

[50] J. T. Batina, AIAA J. 28, 1381 (1990).

[51] A. Rätz and M. Röger, J. Math. Biol. 65, 1215 (2012).

[52] A. Rätz and M. Röger, arXiv:1305.6172v1 (2013). 